OPEN ACCESS

Edited by:

Dieter Steinhilber,

Goethe University Frankfurt, Germany

Reviewed by:

Hua Chen,

Guangxi Normal University, China

Soon Yew Tang,

University of Pennsylvania,

United States

Jun Yang,

China Three Gorges University, China

${ }^{*}$ Correspondence:

Kenneth $\mathrm{K} W \mathrm{U}$

kkgo@nhri.org.tw

Specialty section:

This article was submitted to

Inflammation Pharmacology,

a section of the journal

Frontiers in Pharmacology

Received: 16 August 2021

Accepted: 28 October 2021

Published: 11 November 2021

Citation:

Wu KK (2021) Control of Tissue Fibrosis by 5-Methoxytryptophan, an Innate Anti-Inflammatory Metabolite.

Front. Pharmacol. 12:759199.

doi: 10.3389/fphar.2021.759199

\section{Control of Tissue Fibrosis by 5-Methoxytryptophan, an Innate Anti-Inflammatory Metabolite}

\author{
Kenneth $K W u^{1,2 *}$ \\ ${ }^{1}$ Institute of Cellular and System Medicine, National Health Research Institutes, Zhunan, Taiwan, ${ }^{2}$ Institute of Biotechnology, \\ College of Life Science, National Tsing-Hua University, Hsinchu, Taiwan
}

Tissue fibrosis causes debilitating human diseases such as liver cirrhosis, heart failure, chronic kidney disease and pulmonary insufficiency. It is a dynamic process orchestrated by specific subsets of monocyte-macrophages, fibroblasts, pericytes and hepatic stellate cells. Fibrosis is linked to tissue inflammation. Pro-inflammatory macrophages promote fibrosis by driving myofibroblast differentiation and macrophage myofibroblast transition. Myofibroblasts express $\alpha$-smooth muscle cell actin ( $\alpha-S M A)$ and secrete extracellular matrix (ECM) proteins notably collagen I and III. Deposition of ECM proteins at injury sites and interstitial tissues distorts normal structure and impairs vital functions. Despite advances in the mechanisms of fibrosis at cellular, molecular and genetic levels, prevention and treatment of fibrotic diseases remain poorly developed. Recent reports suggest that 5-methoxytryptophan (5-MTP) is effective in attenuating injury-induced liver, kidney, cardiac and pulmonary fibrosis. It inhibits macrophage activation and blocks fibroblast differentiation to myofibroblasts. Furthermore, it inhibits hepatic stellate cell differentiation into myofibroblasts. As 5-MTP is an endogenous molecule derived from tryptophan catabolism via tryptophan hydroxylase pathway, it is well-suited as a lead compound for developing new anti-fibrotic drugs. This article provides an overview of 5MTP synthesis, and a critical review of its anti-fibrotic activities. Its mechanisms of actions and potential therapeutic value will be discussed.

Keywords: fibrosis, 5-methoxytryptophan, liver cirrhosis, heart failure, fibroblasts, myofibroblasts, hepatic stellate cells

\section{INTRODUCTION}

Fibrous deposition at the injured tissue is a fundamental repair process (Eming et al., 2014). However, uncontrolled fibrous deposition at the injured and normal interstitial tissues leads to structural remodeling and functional defects (Henderson et al., 2020). Fibrosis of diverse human organs including liver, heart, kidney and lung causes debilitating diseases with considerable

\footnotetext{
Abbreviations: $\alpha$-SMA, $\alpha$-smooth muscle actin; ECM, extracellular matrix; MMT, macrophage myofibroblast transition; 5-MTP, 5-methoxytryptophan; COX-2, cyclooxygenase-2; Trp, tryptophan; TPH, tryptophan hydroxylase; ECs, endothelial cells; 5-HTP, 5-hydroxytryptophan; ASMT, acetylserotonin O-methyltransferase; iNOS, inducible nitric oxide synthase; HAT, histone acetyltransferase; LAD, left anterior descending artery occlusion; UUO, unilateral urethral obstruction; DAMP, damage-associated molecular patterns; MSC, mesenchymal stromal cell; ETC, electron transport chain; ROS, reactive oxygen species; Prdx, peroxiredoxins; MKK6, MAP kinase 6; EMT, epithelial mesenchymal transition.
} 
morbidity and mortality. Fibrosis was previously considered to be a passive event. Recent studies with cellular tracking, gene expression profiling and single cell analysis shed lights on the dynamic nature of fibrosis (Wynn and Vannella, 2016). Tissue injury by diverse insults leads to recruitment of pro-inflammatory and pro-fibrotic cells to the injury sites where they undergo phenotypic, transcriptional and metabolic changes to promote fibroblast differentiation into myofibroblasts (Gibb et al., 2020). Myofibroblasts express $a$-smooth muscle actin ( $\alpha$-SMA) and secrete extracellular matrix (ECM) proteins notably collagen I and III and fibronectin (Gibb et al., 2020). ECM proteins deposit at the injured sites and the adjacent interstitial space and distort normal structure and impair organ function. Extensive investigations of the pathological features and molecular mechanisms of fibrosis have shown that despite distinct structural and functional characteristic of the vital organs, fibrosis of liver, heart, kidney and lung shares common pathological, cellular and molecular features. Inflammation is a common hallmark of fibrotic disorders. In fact, fibrosis occurs in inflammatory microenvironment which is enriched with macrophage infiltration (Misharin et al., 2017). Macrophages release myriad pro-inflammatory cytokines and chemokines to elicit inflammatory responses and cause tissue damage. Furthermore, they crosstalk with fibroblasts and promote fibroblasts differentiation to myofibroblasts (Karlmark et al., 2009; Murray et al., 2011; Tang et al., 2019; Zhang F. et al., 2020). Macrophages may be transdifferentiated into myofibroblasts through macrophage myofibroblast transition (MMT) (Murray et al., 2011).

Under normal conditions, resident fibroblasts are quiescent. Upon activation by factors released during tissue injury, they undergo phenotypic switch accompanied by metabolic and transcriptional reprogramming (Gibb et al., 2020). They are the major source of myofibroblasts and the key effector of organ fibrosis. Advances in single cell analytic techniques coupled with RNA sequencing and transcriptomes, have shed lights on heterogeneity of resident fibroblasts in various organs and the involvement of a subset of fibroblasts destined to be differentiated into myofibroblasts (Dobie et al., 2019; Krenkel et al., 2019; Ramachandran et al., 2019; Reyfman et al., 2019). Recent studies using single cell analytic techniques to explore the fibroblast mystery identities a common principle of pathological fibrosis: activation of a selective group of fibroblasts which undergo progressive transcriptional reprogramming and stepwise differentiation into myofibroblasts to generate fibrosis.

Despite molecular and cellular advances in the understanding of fibrosis, there is a gap in prevention and treatment of this devastating human disorder. Several recent reports suggest that 5methoxytryptophan (5-MTP) is effective in attenuating cardiac, renal, hepatic and pulmonary fibrosis in animal models (Figure 1). 5-MTP was discovered in Wu's laboratory as a cyclooxygenase-2 (COX-2) suppressing factor (Deng et al., 2002). It was originally named cytoguardin as it was thought to protect tissues from inflammatory damage (Deng et al., 2002; $\mathrm{Wu}, 2021)$. Metabolomic analysis coupled with genetic approaches identified cytoguardin as a tryptophan (Trp) metabolite, 5-MTP (Cheng et al., 2012). 5-MTP was shown to

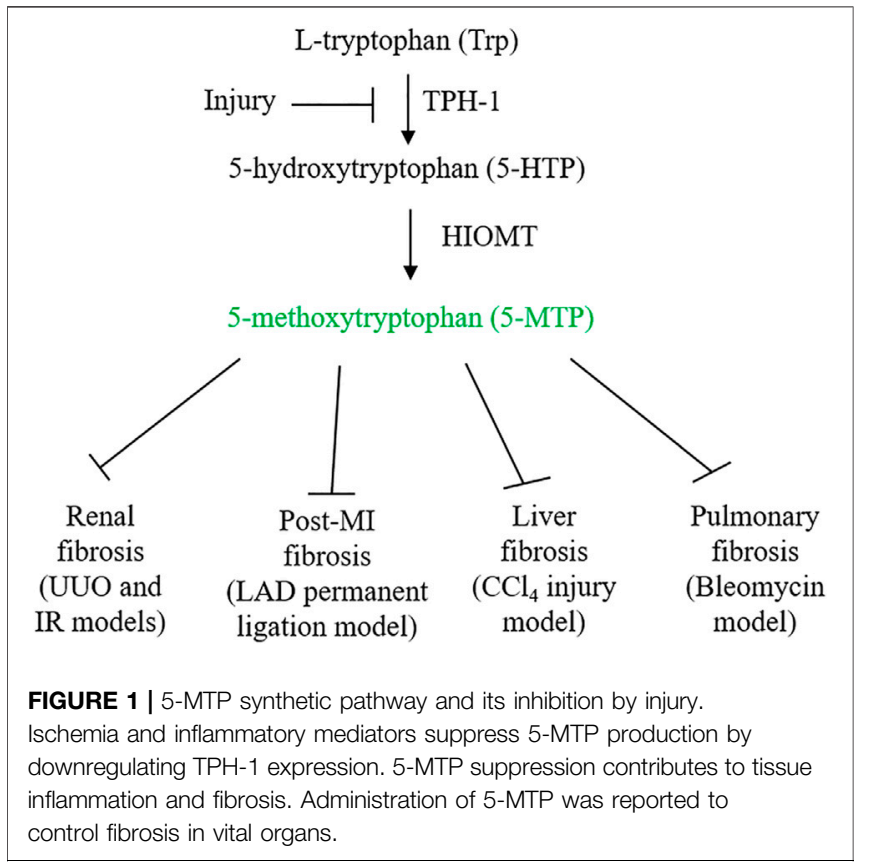

be derived from the tryptophan hydroxylase (TPH) pathway (Cheng et al., 2012). It is produced in fibroblasts, vascular endothelial cells (ECs) and smooth muscle cells, renal and bronchial epithelial cells (Wang et al., 2016). Vascular EC releases 5-MTP into the extracellular milieu via Golgi vesicular trafficking (Wang et al., 2016), and is a major cellular source of blood 5-MTP. 5-MTP was reported to protect endothelial barrier function, control endothelial expression of adhesion molecules and inhibit monocyte/macrophage transmigration (Chu et al., 2016; Wang et al., 2016). Furthermore, 5-MTP inhibits macrophage activation and blocks macrophage release of proinflammatory cytokines and chemokines and expression of COX2 (Wang et al., 2016). These findings indicate that 5-MTP is an innate anti-inflammatory molecule (Wu et al., 2020). As vascular EC is a major cellular source of circulating 5-MTP, 5-MTP is considered to be a new endothelial arsenal against inflammation (Durán and Sánchez, 2016). In view of its efficacy in controlling myofibroblast differentiation and pathological fibrosis in vital organs, 5-MTP has the potential to fulfill the therapeutic gap. The purpose of this paper is to review the anti-fibrotic and antiinflammatory actions of 5-MTP and comment on the mechanisms of actions and the use of 5-MTP as a lead compound in developing new anti-fibrotic drugs.

\section{5-MTP BIOSYNTHESIS IS CATALYZED BY TPH-1 AND HYDROXYINDOLE O-METHYLTRANSFERASE (HIOMT)}

5-MTP is produced from Trp via two enzymatic steps: TPH catalyzes the conversion of Trp to 5-hydroxytryptophan (5-HTP) and HIOMT, conversion of 5-HTP to 5-MTP (Cheng et al., 2012) (Figure 1). With respect to TPH, two isoforms were identified 
and characterized (Boularand et al., 1990; Walther et al., 2003). TPH-2 is expressed in neuronal and pineal cells while TPH-1 is expressed in peripheral tissues. 5-MTP producing cells such as fibroblasts and vascular endothelial cells express only TPH-1. As knockdown of TPH-1 with siRNA abrogates 5-MTP production in fibroblasts, TPH-1 is the isoform catalyzing 5-MTP production (Cheng et al., 2012). Examination of TPH-1 deleted mice has linked TPH-1 to cardiac function: genetic deletion of TPH-1 leads to cardiac failure in mice (Côté et al., 2003). Polymorphism of $T P H-1$ was identified in humans which was considered to be linked to cardiac dysfunction (Lai et al., 2005). However, there has not been reports directly linking TPH-1 polymorphism to cardiac fibrosis or functional impairment. By contrast, it has been reported that TPH-1 expression is suppressed by proinflammatory cytokines which results in reduced 5-MTP production and endothelial dysfunction (Wang et al., 2016; Chu et al., 2016). Furthermore, TPH-1 expression was suppressed in ischemic cardiac and renal tissues which was associated with decreased 5-MTP level, cardiac inflammation and fibrosis and functional impairment (Chen CH. et al., 2019; Hsu et al., 2021) (Figure 1). Thus, TPH-1 defends against inflammation, fibrosis and tissue damage via 5-MTP production. HIOMT was originally detected in pineal cells as the final enzymatic step in melatonin synthesis (Axelrod and Weissbach, 1960, 1961). As it catalyzes conversion of $\mathrm{N}$-acetylserotonin to $\mathrm{N}$-acetyl-5methoxytryptamine (melatonin), it is also called acetylserotonin O-methyltransferase (ASMT). ASMT is a single gene product but three mRNA isoforms due to alternative splicing were detected in pineal cells (Rodriquez et al., 1994; Donohue et al., 1993). Isoform 345 was reported to be the active isoform in melatonin synthesis (Botros et al., 2013). 5-MTP producing cells express only ASMT298 isoform which was reported to be catalytically active in 5-MTP synthesis (Chen et al., 2018). ASMT contains polymorphism in the promoter region which affects ASMT expression and was reported to be a risk factor of autism spectrum disorders (Melke et al., 2008). Several exon mutations affecting ASMT activity were reported which may be associated with sleep disorder (Pagan et al., 2011). Association of ASMT polymorphism/mutation with tissue fibrosis has not been reported. Further work to characterize the relationship may yield valuable information regarding the roles of TPH-1 and HIOMT in inflammation and fibrosis.

\section{5-MTP CONTROLS SYSTEMIC INFLAMMATION}

5-MTP defends against systemic inflammation as reported in a LPS-induced sepsis murine model (Wang et al., 2016). Mice receiving LPS injection develop symptoms and signs mimicking human sepsis. They exhibit surge of circulating cytokines and chemokines ("cytokine storm"), which is accompanied by macrophage infiltration in multiple organs. The infiltrated macrophages express COX-2 and inducible nitric oxide synthase (iNOS) and release abundant cytokines and chemokines. Activated macrophages are responsible not only for organ inflammation but also for cytokine storm. Wang et al. were the first to report that intraperitoneal administration of 5-MTP blocks macrophage expression of COX-2 and release of cytokines in LPS-treated mice. It attenuates macrophage accumulation in lungs and reduces inflammatory response in spleen (Wang et al., 2016). Importantly, it ameliorates sepsis manifestations and reduces sepsis-mediated mortality (Wang et al., 2016).

5-MTP exerts anti-inflammatory effects by targeting activated macrophages. Furthermore, in vitro and in vivo studies suggest that 5-MTP controls macrophage activation by inhibiting p38 MAPKmediated NF-kB activation (Wang et al., 2016). It has also been reported that 5-MTP inhibits transcription co-activator p300 binding and histone acetyltransferase (HAT) activity (Chen et al., 2012; Wang et al., 2016). p300 is a master mediator of proinflammatory gene expression (Grunstein, 1997; Goodman and Smolik, 2000; Thompson et al., 2004). It binds to promoterbound transactivators and bridges this message with transcriptional machinery (Grunstein, 1997; Goodman and Smolik, 2000; Thompson et al., 2004). p300 HAT acetylates histone to open up chromatin structure thereby facilitating transactivator binding (Grunstein, 1997; Goodman and Smolik, 2000; Thompson et al., 2004). It acetylates a large group of transactivators to promote their transcriptional activation of proinflammatory genes including COX-2, iNOS and pro-inflammatory cytokines and chemokines (Deng et al., 2003; Deng et al., 2004). Thus, by interfering with p300 binding and p300 HAT activity, 5MTP is effective in inhibiting the activity of key transactivators such as NF- $\kappa B, C / E B P \beta, A P-1$ and CREB and attenuating expression of pro-inflammatory genes mediated by those transactivators.

\section{5-MTP PROTECTS AGAINST POST-INFARCT MYOCARDIAL FIBROSIS}

Following coronary artery occlusion, ischemia and/or ischemiareperfusion injury induces cardiomyocyte necrosis and apoptosis (Cheng et al., 1996; Olivetti et al., 1996). The damaged cells release chemotactic factors such as CXCL2 and CCL2 which recruit blood monocytes to the injured sites (Deshmane et al., 2009; Guo et al., 2020). Monocytes are differentiated into macrophages, which along with resident macrophages infiltrate the injured and adjacent normal tissues and elicit inflammatory responses. They crosstalk with fibroblasts and induce myofibroblast differentiation. Myofibroblasts drive cardiac fibrosis by release of collagen and an array of extracellular matrix proteins (van den Borne et al., 2010; Kramann et al., 2013; Duffield et al., 2013). Macrophage-mediated inflammatory tissue damage and myocardial fibrosis are cardinal pathophysiological processes causing post-MI myocardial structural changes, functional impairment and heart failure (Prabhu and Frangogiannis, 2016; Talman and Ruskoaho, 2016; Kingery et al., 2017; Bernard et al., 2018). Hsu et al. have recently reported that intraperitoneal administration of 5-MTP in a left anterior descending artery occlusion (LAD) rat model (De Villiers and Riley, 2020) reduces macrophage infiltration, attenuates myocardial fibrosis and preserves ventricular structure and function (Hsu et al., 2021). They first determined route, timing and doses of 5-MTP administration, 


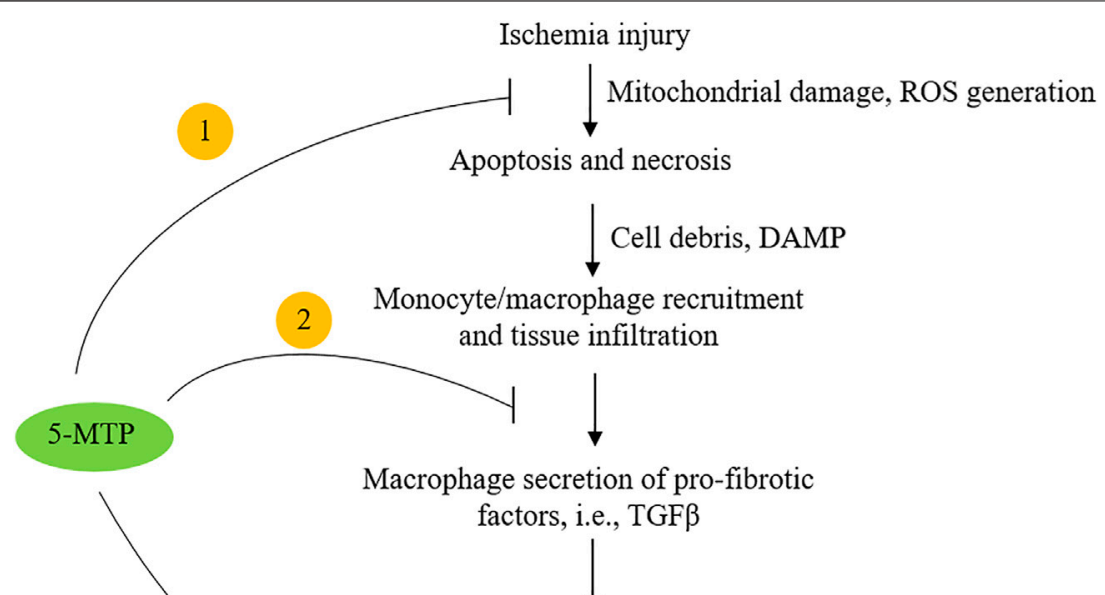

Fibroblast activation and differentiation

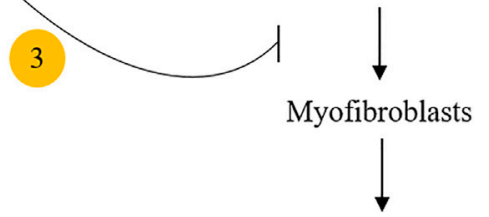

Collagen and other ECM

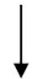

Fibrosis

FIGURE 2 | Highlight of the potential mechanisms by which 5-MTP inhibits cardiac fibrosis. 5-MTP exerts cellular actions by (1) scavenging ROS and reducing apoptosis; (2) blocking macrophage secretion of TGF $\beta$ and other pro-fibrotic factors and (3) reducing myofibroblasts.

and found that administration of two doses of 5-MTP at $17 \mathrm{mg} / \mathrm{kg}$ within $24 \mathrm{~h}$ after LAD occlusion optimally reduced inflammation and fibrosis and preserved structural integrity and ventricular function (Hsu et al., 2021). Additional dosing after the $24 \mathrm{~h}$-window did not provide additional advantage. At $48 \mathrm{~h}$ after LAD occlusion, there was a significant reduction of macrophages in the infarct and peri-infarct areas in 5-MTPtreated rats when compared to control rats (Hsu et al., 2021). Furthermore, expression of IL-1 $\beta$, IL-6, IL-18, CCL2, CXCL2 and CXCL10 was significantly lower in 5-MTP treated than in control rats (Hsu et al., 2021). At day 28 after LAD occlusion, extensive transmural fibrosis and interstitial fibrosis developed which was accompanied by structural remodeling. Intraperitoneal 5-MTP administration within $24 \mathrm{~h}$ of LAD occlusion attenuated cardiomyocyte apoptosis and infarct volume and improved left ventricular function accompanied by reduced fibrosis and transmural scar in the left ventricular region. TGF $\beta$, a master mediator of fibrosis (Leask and Abraham, 2004; Bernard et al., 2018) was increased in infarct zone as a result of macrophage infiltration. Macrophage iNOS expression which was increased in the infarct zone has been implicated in myocardial fibrosis and heart failure (Chen CH. et al., 2019). 5-MTP was shown to reduce TGF $\beta$ level and attenuate iNOS expression in macrophages at the infarct zone (Hsu et al., 2021). These findings suggest that 5-MTP prevents post-MI myocardial fibrosis by controlling ROS generation, inhibiting macrophage activation and suppressing pro-fibrotic gene expression (Figure 2).

\section{5-MTP DEFENDS KIDNEYS AGAINST URETHRAL OBSTRUCTION-INDUCED FIBROSIS}

Metabolomic analysis of serum metabolites in chronic kidney diseases identified 5-MTP as a major metabolite which is inversely associated with progression of kidney diseases (Chen DQ. et al., 2019). In experimental animal models such as unilateral urethral obstruction (UUO) murine model, renal production of 5-MTP was reported to be reduced accompanied by progressive renal damage leading to renal fibrosis and functional impairment. Urethral obstruction elicits renal inflammation and inflammation-mediated tissue damage and fibrosis. It has been shown that renal tubular epithelial cells produce 5-MTP (Wang et al., 2016). It is possible that decrease of renal 5-MTP production following UUO is due to suppression of renal tubular cell TPH-1. Chen et al. reported that intraperitoneal administration of 5-MTP rescues kidney from UUO-induced renal inflammation and fibrosis (Chen CH. et al., 2019). 5-MTP administration reduced macrophage infiltration and proinflammatory gene expression. 5-MTP downregulated 
expression of pro-fibrotic genes suggesting that 5-MTP prevents myofibroblast differentiation in injured kidneys. In vitro epithelial cell experiments reveal that TGF $\beta$ and LPS induce expression of pro-fibrotic factors accompanied by suppression of TPH-1 expression and 5-MTP production. Pretreatment with 5-MTP rescues renal epithelial cells from TGF- $\beta$ - and LPS-induced proinflammatory and pro-fibrotic phenotypic switch. Furthermore, 5MTP blocks NF- $\kappa \mathrm{B}$ activation induced by LPS. TPH-1 overexpression in renal epithelial cells restores 5-MTP production and renders epithelial cells resistant to LPS- and TGF $\beta$-induced pro-fibrotic and pro-inflammatory changes. Renal ischemia-reperfusion injury results in chronic inflammatory and profibrotic pathophysiological changes resembling ischemia-reperfusion injury to the heart. 5-MTP attenuates ischemia-reperfusion induced renal inflammation and fibrosis in a fashion similar to protection of heart from inflammatory tissue damage and fibrosis (Chen DQ. et al., 2019).

Following UUO or ischemia-reperfusion injury, several cell types were considered to be the source of myofibroblasts (Duffield, 2014; Wouters et al., 2015). Recent studies using single cell RNA sequencing and spatial transcriptomics, have shown that myofibroblasts are derived from resident fibroblasts and pericytes (Kuppe et al., 2021). It remains to be investigated whether 5-MTP has a direct effect on the fibrogenic cells.

\section{5-MTP SUPPRESSES HEPATIC STELLATE CELL ACTIVATION AND TRANSDIFFERENTIATION}

Uncontrolled liver fibrosis leads to debilitating liver cirrhosis and liver failure. Liver fibrosis is a common pathological event induced by viral infections, chronic alcoholism, non-alcoholic liver disease and non-alcoholic steatohepatitis. These pathophysiological conditions are associated with release of myriad factors to activate HSCs (Tsuchida and Friedman, 2017). HSCs are localized at the junction of sinusoid endothelial cells and hepatocytes. They represent a major cell type in liver: approximately $10 \%$ of liver cells are HSCs. At resting state, they are quiescent. Upon liver injury, damaged hepatocytes release pro-fibrotic factors and generate ROS and damageassociated molecular patterns (DAMP), which activate HSCs and induce their differentiation into myofibroblasts (Du et al., 2018; Khomich et al., 2019; An et al., 2020). It has been shown that activated HSCs exhibit major changes in energy metabolism shifting from oxidative phosphorylation to aerobic glycolysis and glutaminolysis (Du et al., 2018; Khomich et al., 2019). Mitochondrial TCA cycle is interrupted and metabolites such as succinate are accumulated and released. Extracellular succinate induces HSC differentiation through binding to a specific succinate receptor, SUCNR-1 (also known as GPR91) (Li et al., 2015; Li et al., 2016; Cho, 2018).

5-MTP was reported to attenuate $\mathrm{CCl} 4$-induced liver cirrhosis in a murine model. 5-MTP at a relatively low dose $(5 \mathrm{mg} / \mathrm{kg}$ twice weekly for 8 weeks) was effective in reducing a-SMA expressing cells and attenuating liver fibrosis (Tong et al., 2021). In the in vitro experiments with cultured HSC, LX-2 cells, 5-MTP
Liver injury with carbon tetrachloride

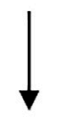

Hepatocyte damage and death

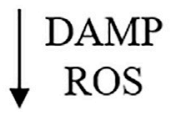

Activation of hepatic stellate cells

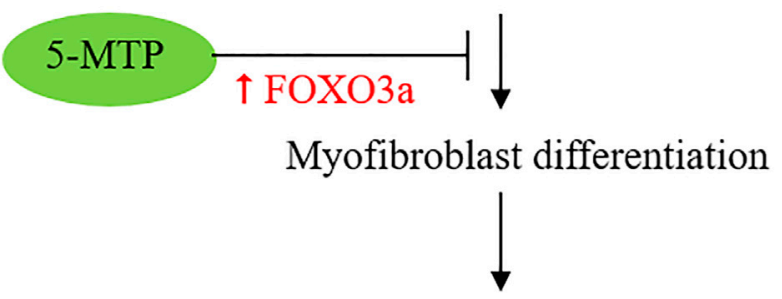

ECM deposition

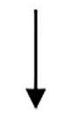

Liver fibrosis

FIGURE 3 | Schematic illustration of the effect of 5-MTP on attenuating $\mathrm{HSC}$ differentiation to myofibroblasts via $\mathrm{FOXO}$ a upregulation. $\mathrm{FOXO}$ a was reported to suppress miR21 expression and thereby restore ATG5 and ATG5mediated autophagy.

suppressed TGF $\beta$-induced expression $\alpha$-SMA, fibronectin, collagen I and III, suggesting that 5-MTP may block differentiation of HSCs into myofibroblasts. Tong et al. have shown that 5-MTP blocks the pro-fibrotic property of HSC by regulating FOXO3a/miR21/ATG5 pathway (Tong et al., 2021). They have provided experimental evidence that miR21 binds and inhibits ATG5 and thereby controls autophagy. FOXO3a inhibits miR21 expression and restores ATG5 for participation in autophagy. Autophagy was reported to reduce hepatic fibrosis (Zhu et al., 1999; Hidvegi et al., 2010). However, other reports suggest autophagy activates HSC and promotes hepatic fibrosis (Hernández-Gea et al., 2012). The complex role of autophagy in hepatic fibrosis may be due to different cell types, stimuli and experimental conditions (Mao and Fan, 2015; Li et al., 2020). By using genetic knockdown and pharmacological inhibition, Tong et al have shown that 5-MTP inhibits HSC proliferation and differentiation by upregulating $\mathrm{FOXO} 3 \mathrm{a}$ and restoration of autophagy. This observation was consistent with previous reports that 5-MTP prevents mesenchymal stromal cell (MSC) senescence by upregulation of FOXO3 (Chang et al., 2017) and that FOXO3 plays a pivotal role in controlling fibrosis (Al-Tamari et al., 2018). Taken together, the reported findings suggest that 5 MTP attenuates liver fibrosis by upregulating FOXO3a (Figure 3) and the consequent suppression of miR21 expression. It is to be noted that effective 5-MTP concentrations to prevent MSC 
senescence are one to two orders of magnitude higher than those used in blocking HSC activation and differentiation. Further studies are needed to clarify the dosing difference.

\section{5-MTP INHIBITS BLEOMYCIN-INDUCED PULMONARY FIBROSIS}

Pulmonary alveolar epithelial cells are vulnerable to injury by environmental toxins, drugs, radiation and immune attacks. Uncontrolled damage leads to cell loss and fibrosis (Noble et al., 2012; Wuyts et al., 2013). Pulmonary fibrosis destroys normal alveolar structure and impairs lung function resulting in chronic debilitating illness (Sgalla et al., 2019). Recent advances in the cellular and molecular mechanisms of lung fibrosis provide evidence to indicate that pulmonary fibrosis shares with fibrosis of other organs key pathological features such as recruitment of monocytes to promote fibrosis, conversion of resident fibroblasts to myofibroblasts and activation of pro-fibrotic transcriptional programs (Zepp et al., 2017; Peyser et al., 2019). It is thus not unexpected that 5-MTP inhibits bleomycin-induced pulmonary fibrosis in a murine model (Fang et al., 2020). In this model, Fang et al. showed that 5-MTP administration to bleomycin-treated mice reduced collagen deposition, myofibroblasts accumulation and alveolar architectural destruction. It disrupted TGF $\beta$ / SMAD3 and PI-3K/Akt pathways. In vitro experiments provide evidence to support the observation that 5-MTP inhibits fibroblast differentiation to myofibroblasts and reduces fibroblast migration by blocking TGF $\beta$ signaling pathway (Fang et al., 2020). These findings suggest that 5-MTP is effective in attenuating pulmonary fibrosis due to external injury. It is unclear whether 5-MTP exerts a similar effect on idiopathic pulmonary fibrosis, the most common and the most severe form of human pulmonary interstitial fibrotic diseases (Martinez et al., 2005).

\section{POTENTIAL MECHANISMS BY WHICH 5-MTP CONTROLS FIBROSIS}

The exact mechanisms by which 5-MTP reduces fibrosis in multiple organs are not completely understood and remain to be investigated. However, recent reports suggest that 5-MTP exerts its anti-fibrotic actions by controlling multiple steps of pro-fibrotic cellular changes, transcriptional reprogramming and signaling pathways (Figure 4).

\section{5-MTP Inhibits Ischemia-Induced Apoptosis}

Immediately following coronary artery occlusion, hypoxia induces rapid metabolic changes. Glycolysis is enhanced while oxidative phosphorylation is shut down, resulting in TCA cycle interruption and accumulation of succinate (Chouchani et al., 2014; Zhang et al., 2018). Furthermore, mitochondrial electron transport chain (ETC) becomes disorganized with a reverse electron transport resulting in generation of reactive oxygen species (ROS) (Chouchani et al., 2014). Balance of mitochondrial dynamics (mitochondrial fusion vs. fission) is altered to favor mitochondrial fission resulting in mitochondrial fragmentation

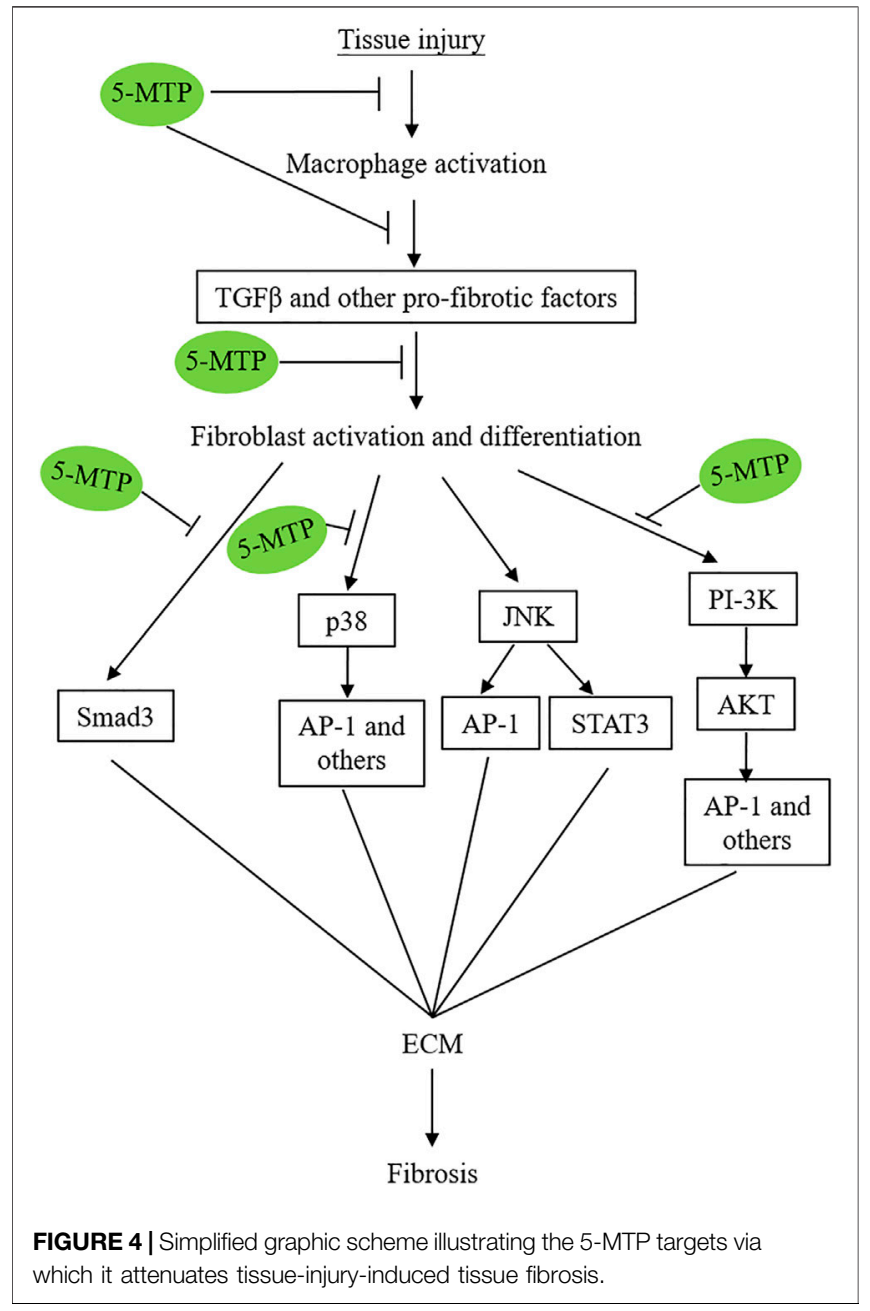

which is accompanied by uncontrolled mitochondrial membrane permeability resulting in cellular necrosis and apoptosis (Ong et al., 2010; Ramachandra et al., 2020). Massive cardiomyocyte death creates an infarct zone. Damaged and dead heart cells produce chemotactic factors to recruit neutrophils, monocytes and lymphocytes from circulating blood to the infarct and peri-infarct zone. DAMP as well as cell debris and mitochondrial DNA is generated to activate the recruited blood cells and elicit inflammatory responses (Ramachandran et al., 2012; Mouton et al., 2018). Excessive inflammation induces additional cardiac cell damage and expand the infarct size and triggers the subsequent tissue fibrosis. Reported data indicate that 5-MTP attenuates apoptosis and reduces infarct size following LAD occlusion in the rat model (Hsu et al., 2021). 5-MTP reduces caspase 3 level of myocardial tissues and caspase 3-positive cardiomyocytes at the peri-infarct region (Hsu et al., 2021). These findings suggest that 5-MTP reduces mitochondrial damage and controls mitochondrial release of cytochrome C. Furthermore, 5MTP preserved TOM20, a mitochondrial outer membrane protein, which is decreased by ischemia, suggesting that 5-MTP maintains mitochondrial membrane integrity during ischemic attacks and thus attenuates apoptosis via the mitochondrial-dependent pathway (Hsu 
et al., 2021). Inhibition of ischemia- and toxin-induced mitochondrial damage and the consequent release of proapoptotic factors from mitochondria is a major attribute by which 5-MTP attenuates inflammation and fibrosis. This event represents an early window of opportunity for fibrosis control.

\section{5-MTP Scavenges ROS and Prevents ROS-Induced Cell Death and Senescence}

During tissue injury by ischemia, toxins and chemicals, there is continued generation of ROS: initially via damaged mitochondria followed by activation of cytosolic NADPH oxidase and xanthine oxidase (Chouchani et al., 2016; Zhang Y. et al., 2020). ROS are scavenged by mitochondrial antioxidant enzymes notably MnSOD and peroxiredoxins (Prdx) (Cox et al., 2009) and cytosolic antioxidant enzymes such as catalase. ROS induce a broad spectrum of pathological changes including cell deaths, inflammation and fibrosis (Gonzalez-Gonzalez et al., 2017; Moris et al., 2017). It was reported that ROS promote fibrosis by enhancing TGF $\beta$ signaling (Gonzalez-Gonzalez et al., 2017). 5MTP was reported to reduce ROS generation in mesenchymal stromal cells induced by high glucose by upregulating MnSOD and catalase activities (Chang et al., 2017). Hsu et al. reported in the rat MI model that 5-MTP administration within $24 \mathrm{~h}$ after LAD occlusion reduced accumulation of ROS (Hsu et al., 2021). Their results reveal that 5-MTP restored MnSOD and Prdx3 and inhibited NADPH oxidase by suppressing expression of NOX2 and NOX4 (Hsu et al., 2021).

\section{5-MTP Controls the Transcriptional Network That Drives Fibrosis}

The subset of fibroblasts, which are destined to be differentiated to myofibroblasts exhibit distinct transcriptional network. Several transcriptional activators have been identified as master driver of fibroblast commitment. PU.1 was reported to drive fibroblast polarization and to be essential for tissue fibrosis (Wohlfahrt et al., 2019). Inhibition of PU.1 prevents bleomycin-induced skin fibrosis. AP-1 transactivator plays a pivotal role in organ fibrosis. Activation of c-Jun, a subunit of AP-1, was sufficient to induce diverse fibrotic diseases (Wernig et al., 2017; Schulien et al., 2019). AP-1 binds to the promoter region of profibrotic and proinflammatory genes including collagen and ECM proteins, growth factors, cytokines and COX-2 (Manabe et al., 2002), which contribute to cardiac and pulmonary fibrosis (Rajasekaran et al., 2013). Other transactivators such as NF$\kappa \mathrm{B}, \mathrm{C} / \mathrm{EBP} \beta$ and $\mathrm{CREB}$ were reported to be activated in fibrotic tissues and contribute to fibrosis. For example, NF- $\kappa B$ has been shown to regulate activation of HSCs and hepatic myofibroblast differentiation (Luedde and Schwabe, 2011) and promote cardiac remodeling (Hamid et al., 2011). C/EBP $\beta$ phosphorylation at Thr 217 contributes to lung fibrosis in mice (Buck and Chojkier, 2011) and mesenchymal specific deletion of $\mathrm{C} / \mathrm{EBP} \beta$ suppresses pulmonary fibrosis (Hu et al., 2012).

5-MTP was discovered as a suppressor of COX-2 expression (Deng et al., 2002; Cheng et al., 2012). It inhibits COX-2 transcription by inhibiting binding of NF- $\kappa \mathrm{B}, \mathrm{AP}-1, \mathrm{C} / \mathrm{EBP} \beta$ and CREB to COX-2 promoter (Chen et al., 2012). As COX-2 is considered to be a prototypic immediate early gene and shares with pro-inflammatory cytokines, adhesion molecules and profibrotic genes common promoter characteristics, it is likely that 5MTP attenuates injury-induced organ fibrosis through blocking activation and binding of AP-1 and other transactivators to the promoter regions of pro-fibrotic genes. p300 inhibitor was reported to ameliorate cardiac and renal fibrosis (Rai et al., 2017) indicating that p300 is intimately involved in profibrotic transcriptional program. 5-MTP inhibits p300 binding and HAT activity via which it exerts potent actions on controlling transcription of pro-inflammatory and pro-fibrotic genes.

\section{5-MTP Disrupts Pro-Fibrotic Signaling Pathways}

A number of signaling pathways including p38 MAPK (Ma et al., 1999; Stambe et al., 2004; Molkentin et al., 2017; Turner and Blythe, 2019), TGF $\beta /$ SMAD3 (Fang et al., 2020), PI3K/AKT (Zhang et al., 2016; Hsu et al., 2017; Fang et al., 2020; Hu et al., 2020); JNK (Grynberg et al., 2017) and STAT3 (Chakraborty et al., 2017) were reported to mediate tissue fibrosis. p38 MAPK plays a central regulatory role. Molkentin et al. have used transgenic approaches to demonstrate that fibroblast-specific transgenic overexpression of MAP kinase 6 (MKK6), an upstream inducer of p38 MAPK, results in interstitial and perivascular fibrosis in the heart, lung and kidney accompanied by increased myofibroblast (Molkentin et al., 2017). Genetic deletion of Mapk14 (coding for p38 MAPK) blocks fibroblast differentiation to myofibroblasts in a murine cardiac injury model (Ma et al., 1999). These results suggest that p38 MAPK is a major signaling molecule of fibroblast differentiation and activation and plays a central role in in pathological fibrosis.

TGF $\beta$ induces fibroblast activation and myofibroblast differentiation (Froese et al., 2016; Meng et al., 2016) by binding to a membrane heterodimeric receptor which activate and differentiate fibroblasts via several signaling pathways (Luo and Lodish, 1996). The canonical pathway is mediated via SMAD3 which has been shown to be critical for myofibroblast differentiation (Hu et al., 2007; Dobaczewski et al., 2010). TGF $\beta$ may induce fibroblast activation and myofibroblast differentiation via other signaling pathways independent of SMAD3 among which p38 MAPK pathway and PI-3K/AKT are well characterized non-canonical signaling pathways. Furthermore, it has been reported that p38 MAPK mediates fibrogenic signal through SMAD3 photophosphorylation suggesting a crosstalk between p38 MAPK and SMAD (Furukawa et al., 2003). It has been suggested that various signaling pathways converge at STAT-3 to mediate TGF $\beta$ induced tissue fibrosis (Chakraborty et al., 2017).

5-MTP was reported to block p38 MAPK activation in a number cellular models under different stresses. For example, it was reported that 5-MTP protects endothelial VE-cadherin and therefore endothelial barrier function by blocking p38 MAPK activation (Chu et al., 2016). 5-MTP suppresses cytokine-induced vascular SMC phenotypic switch by blocking p38 MAPK and 
thereby maintaining contractile vascular SMC contractile phenotype (Chen CH. et al., 2019). Importantly, 5-MTP inhibits macrophage activation and secretion of cytokines by inhibiting p38 MAPK signaling pathway (Wang et al., 2016). An additional supportive evidence is derived from cancer cell epithelial mesenchymal transition (EMT) (Cheng et al., 2016). EMT is an important cellular phenotypic switch via which cancer cells achieve metastatic characteristics. It was reported that 5MTP was effective in reducing A549 cancer cell EMT via suppressing p38 MAPK (Cheng et al., 2016). Taken together, the reported data suggest that 5-MTP exerts its multiple protective effects through blocking p38 MAPK activation and p38 MAPK signaling pathways.

Fang et al. reported that 5-MTP attenuates pulmonary fibrosis by blocking TGFß-induced SMAD3 and PI3K/AKT signaling pathways (Fang et al., 2020). Thus, 5-MTP controls tissue fibrosis by inhibiting several pro-fibrotic signaling pathways (Figure 4). Of note, these signaling pathways cross-talk to promote fibroblast activation, differentiation and fibrosis.

It remains to be determined how 5-MTP blocks diverse signaling pathways. Preliminary data suggest that 5-MTP acts via a specific membrane receptor (Wang et al., 2018). It is possible that 5-MTP blocks p38 MAPK, SMAD3 and PI-3K/AKT through cross-talk between the signaling pathway mediated by 5-MTP receptor activation and stress-induced pro-fibrotic signaling pathways. As 5-MTP receptors have not been identified and characterized, the potential cross-talk is hypothetical and requires further validation.

\section{CONCLUSION}

Vascular endothelial cells, renal and pulmonary epithelial cells, fibroblasts and cardiomyocytes produce 5-MTP which defends vital organs against injury and maintains tissue homeostasis. Proinflammatory mediators suppress 5-MTP production through inhibiting TPH-1 expression, and disrupting the homeostatic balance leading to tissue damage and fibrosis. Supplementation with exogenous 5-MTP rescues tissues from inflammatory damage and prevents fibrosis and structural remodeling. 5-MTP was recently reported to protect against post-infarct cardiac fibrosis and left ventricular remodeling in a rat LAD permanent ligation model (Hsu et al., 2021). The experimental data suggest that 5MTP exerts anti-fibrotic effects through control of early ROS accumulation, apoptosis and macrophage recruitment. 5-MTP attenuates renal fibrosis and functional failure in a UUO model (Chen DQ. et al., 2019). 5-MTP reduces bleomycin-induced alveolar epithelial cell damage and induces interstitial fibrosis through downregulating TGF $\beta$ and PI-3K signaling pathways (Fang et al., 2020). Finally, 5-MTP was reported to alleviate $\mathrm{CCl}_{4}$-induced liver fibrosis through FoxO3a-mediated autophagy (Tong et al., 2021). These reports indicate that 5MTP possesses universal anti-fibrotic actions and is a potential lead compound for developing new therapy for fibrotic disorder.

5-MTP exerts the anti-fibrotic effect by inhibiting macrophage recruitment and activation, which is closely linked to its antiinflammatory actions. Macrophage infiltration in injured tissues is a cardinal manifestation of tissue inflammation, fibrosis and organ failure. 5-MTP blocks monocyte transmigration and macrophage secretion of chemokines thereby reducing macrophage accumulation at the tissue injured site. Furthermore, 5-MTP inhibits macrophage release of pro-inflammatory cytokines notably IL-1 $\beta$, TNF $\alpha$ and IL- 6 and pro-fibrotic factors such as TGF $\beta$, PDGF and cytokines. Macrophages are heterogenous. Subsets of macrophages are functionally selective for inflammatory responses and fibrotic formation, respectively. It remains to be determined whether 5-MTP possesses selective actions on functionally distinct subsets of macrophages.

5-MTP controls macrophage activation by inhibiting p300 HAT activity and binding of pro-inflammatory transactivators such as NF- $\kappa B, A P-1, C / E B P \beta$ and CREB. Since AP-1 plays a central role in mediating fibrosis, it is likely that 5-MTP inhibits pro-fibrotic gene expressions in fibroblasts and myofibroblasts by blocking the activity and binding of AP-1. p38 MAPK is a key signaling pathway mediating inflammation and fibrosis. 5-MTP is known to inhibit p38 MAPK activation in macrophages, vascular ECs and SMCs. It is likely that 5-MTP inhibits pro-inflammatory transactivators by disrupting p38 MAPK activation and downstream signaling.

The antifibrotic and anti-inflammatory actions of 5-MTP are likely to be mediated by interaction between 5-MTP and its cellular surface receptors. Preliminary data suggest that the 5MTP receptor is a $\mathrm{G}$ protein-coupled receptor. It is possible that signaling from 5-MTP receptors inhibits p38 MAPK activation, and/or upregulating p38 MAPK inhibitors (Kumar et al., 2003).

Development of anti-fibrotic therapy has been hampered by incomplete understanding of the complex cellular and molecular mechanisms underlying initiation and progression of fibrosis. Another hurdle that hampers development of antifibrotic therapy is lack of an effective strategy to block selectively pathological fibrosis while preserving physiological fibrosis, which is vital to tissue repair. 5-MTP represents a new class of compounds that could overcome the obstacles. It offers several advantages. Foremost is that it is an endogenously produced metabolite with known anti-inflammatory and tissue protection properties. Secondly, it suppresses but does not completely abolish macrophage activation and fibroblast differentiation. It is therefore well suited to be a lead compound for developing new anti-fibrotic drugs. 5-MTP derivatives were recently granted multi-national patents. As post-MI heart failure and post-injury chronic kidney diseases emerge as a major disease burden with great health and socioeconomic impacts, 5-MTP derivatives will be a valuable addition to prevention and treatment of those devastating illnesses.

\section{AUTHOR CONTRIBUTIONS}

The author confirms being the sole contributor of this work and has approved it for publication.

\section{FUNDING}

This work was funded by The Excellent Team Research Program Grant from Ministry of Science and Technology, Taiwan (1072321-B-400-011; 108-2321-B-400-008). 


\section{REFERENCES}

Al-Tamari, H. M., Dabral, S., Schmall, A., Sarvari, P., Ruppert, C., Paik, J., et al. (2018). FoxO3 an Important Player in Fibrogenesis and Therapeutic Target for Idiopathic Pulmonary Fibrosis. EMBO Mol. Med. 10, 276-293. doi:10.15252/ emmm.201606261

An, P., Wei, L. L., Zhao, S., Sverdlov, D. Y., Vaid, K. A., Miyamoto, M., et al. (2020). Hepatocyte Mitochondria-Derived Danger Signals Directly Activate Hepatic Stellate Cells and Drive Progression of Liver Fibrosis. Nat. Commun. 11, 2362. doi:10.1038/s41467-020-16092-0

Axelrod, J., and Weissbach, H. (1960). Enzymatic O-Methylation of N-Acetylserotonin to Melatonin. Science 131, 1312. doi:10.1126/ science.131.3409.1312

Axelrod, J., and Weissbach, H. (1961). Purification and Properties of Hydroxyindole-O-Methyl Transferase. J. Biol. Chem. 236, 211-213. doi:10.1016/s0021-9258(18)64458-8

Bernard, K., Logsdon, N. J., Benavides, G. A., Sanders, Y., Zhang, J., Darley-Usmar, V. M., et al. (2018). Glutaminolysis Is Required for Transforming Growth Factor-B1-Induced Myofibroblast Differentiation and Activation. J. Biol. Chem. 293, 1218-1228. doi:10.1074/jbc.RA117.000444

Botros, H. G., Legrand, P., Pagan, C., Bondet, V., Weber, P., Ben-Abdallah, M., et al. (2013). Crystal Structure and Functional Mapping of Human ASMT, the Last Enzyme of the Melatonin Synthesis Pathway. J. Pineal Res. 54, 46-57. doi:10.1111/j.1600-079X.2012.01020.x

Boularand, S., Darmon, M. C., Ganem, Y., Launay, J. M., and Mallet, J. (1990). Complete Coding Sequence of Human Tryptophan Hydroxylase. Nucleic Acids Res. 18, 4257. doi:10.1093/nar/18.14.4257

Buck, M., and Chojkier, M. (2011). C/EBP $\beta$-Thr217 Phosphorylation Signaling Contributes to the Development of Lung Injury and Fibrosis in Mice. PLoS One 6, e25497. doi:10.1371/journal.pone.0025497

Chakraborty, D., Šumová, B., Mallano, T., Chen, C. W., Distler, A., Bergmann, C., et al. (2017). Activation of STAT3 Integrates Common Profibrotic Pathways to Promote Fibroblast Activation and Tissue Fibrosis. Nat. Commun. 8, 1130. doi:10.1038/s41467-017-01236-6

Chang, T. C., Hsu, M. F., Shih, C. Y., and Wu, K. K. (2017). 5-methoxytryptophan Protects MSCs from Stress Induced Premature Senescence by Upregulating FoxO3a and mTOR. Sci. Rep. 7, 11133. doi:10.1038/s41598-017-11077-4

Chen, B. R., Cheng, H. H., Lin, W. C., Wang, K. H., Liou, J. Y., Chen, P. F., et al. (2012). Quiescent Fibroblasts Are More Active in Mounting Robust Inflammatory Responses Than Proliferative Fibroblasts. PLoS One 7, e49232. doi:10.1371/journal.pone.0049232

Chen, C. H., Ho, Y. C., Ho, H. H., Liang, L. Y., Jiang, W. C., Lee, G. L., et al. (2019a). Tryptophan Metabolite 5-Methoxytryptophan Ameliorates Arterial Denudation-Induced Intimal Hyperplasia via Opposing Effects on Vascular Endothelial and Smooth Muscle Cells. Aging (Albany NY) 11, 8604-8622. doi:10.18632/aging.102350

Chen, D. Q., Cao, G., Chen, H., Argyopoulos, C. P., Yu, H., Su, W., et al. (2019b). Identification of Serum Metabolites Associating with Chronic Kidney Disease Progression and Anti-Fibrotic Effect of 5-Methoxytryptophan. Nat. Commun. 10, 1476. doi:10.1038/s41467-019-09329-0

Chen, H. L., Yuan, C. Y., Cheng, H. H., Chang, T. C., Huang, S. K., Kuo, C. C., et al. (2018). Restoration of Hydroxyindole O-Methyltransferase Levels in Human Cancer Cells Induces a Tryptophan-Metabolic Switch and Attenuates Cancer Progression. J. Biol. Chem. 293, 11131-11142. doi:10.1074/jbc.RA117.000597

Cheng, H. H., Chu, L. Y., Chiang, L. Y., Chen, H. L., Kuo, C. C., and Wu, K. K. (2016). Inhibition of Cancer Cell Epithelial Mesenchymal Transition by Normal Fibroblasts via Production of 5-Methoxytryptophan. Oncotarget 7, 31243-31256. doi:10.18632/oncotarget.9111

Cheng, H. H., Kuo, C. C., Yan, J. L., Chen, H. L., Lin, W. C., Wang, K. H., et al. (2012). Control of Cyclooxygenase-2 Expression and Tumorigenesis by Endogenous 5-methoxytryptophan. Proc. Natl. Acad. Sci. U. S. A. 109, 13231-13236. doi:10.1073/pnas.1209919109

Cheng, W., Kajstura, J., Nitahara, J. A., Li, B., Reiss, K., Liu, Y., et al. (1996). Programmed Myocyte Cell Death Affects the Viable Myocardium after Infarction in Rats. Exp. Cel Res. 226, 316-327. doi:10.1006/excr.1996.0232

Cho, E. H. (2018). Succinate as a Regulator of Hepatic Stellate Cells in Liver Fibrosis. Front. Endocrinol. (Lausanne) 9, 455. doi:10.3389/fendo.2018.00455
Chouchani, E. T., Pell, V. R., Gaude, E., Aksentijević, D., Sundier, S. Y., Robb, E. L., et al. (2014). Ischaemic Accumulation of Succinate Controls Reperfusion Injury through Mitochondrial ROS. Nature 515, 431-435. doi:10.1038/nature13909

Chouchani, E. T., Pell, V. R., James, A. M., Work, L. M., Saeb-Parsy, K., Frezza, C., et al. (2016). A Unifying Mechanism for Mitochondrial Superoxide Production during Ischemia-Reperfusion Injury. Cell Metab 23, 254-263. doi:10.1016/ j.cmet.2015.12.009

Chu, L. Y., Wang, Y. F., Cheng, H. H., Kuo, C. C., and Wu, K. K. (2016). Endothelium-Derived 5-Methoxytryptophan Protects Endothelial Barrier Function by Blocking P38 MAPK Activation. PLoS One 11, e0152166. doi:10.1371/journal.pone.0152166

Côté, F., Thévenot, E., Fligny, C., Fromes, Y., Darmon, M., Ripoche, M. A., et al. (2003). Disruption of the Nonneuronal Tph1 Gene Demonstrates the Importance of Peripheral Serotonin in Cardiac Function. Proc. Natl. Acad. Sci. U. S. A. 100, 13525-13530. doi:10.1073/pnas.2233056100

Cox, A. G., Winterbourn, C. C., and Hampton, M. B. (2009). Mitochondrial Peroxiredoxin Involvement in Antioxidant Defence and Redox Signalling. Biochem. J. 425, 313-325. doi:10.1042/BJ20091541

De Villiers, C., and Riley, P. R. (2020). Mouse Models of Myocardial Infarction: Comparing Permanent Ligation and Ischaemia-Reperfusion. Dis. Model. Mech. 13, dmm046565. doi:10.1242/dmm.046565

Deng, W. G., Saunders, M., Gilroy, D., He, X. Z., Yeh, H., Zhu, Y., et al. (2002). Purification and Characterization of a Cyclooxygenase-2 and Angiogenesis Suppressing Factor Produced by Human Fibroblasts. FASEB J. 16, 1286-1288. doi:10.1096/fj.01-0844fje

Deng, W. G., Zhu, Y., and Wu, K. K. (2004). Role of P300 and PCAF in Regulating Cyclooxygenase-2 Promoter Activation by Inflammatory Mediators. Blood 103, 2135-2142. doi:10.1182/blood-2003-09-3131

Deng, W. G., Zhu, Y., and Wu, K. K. (2003). Up-Regulation of P300 Binding and P50 Acetylation in Tumor Necrosis Factor-Alpha-Induced Cyclooxygenase-2 Promoter Activation. J. Biol. Chem. 278, 4770-4777. doi:10.1074/jbc.M209286200

Deshmane, S. L., Kremlev, S., Amini, S., and Sawaya, B. E. (2009). Monocyte Chemoattractant Protein-1 (MCP-1): an Overview. J. Interferon Cytokine Res. 29, 313-326. doi:10.1089/jir.2008.0027

Dobaczewski, M., Bujak, M., Li, N., Gonzalez-Quesada, C., Mendoza, L. H., Wang, X. F., et al. (2010). Smad3 Signaling Critically Regulates Fibroblast Phenotype and Function in Healing Myocardial Infarction. Circ. Res. 107, 418-428. doi:10.1161/CIRCRESAHA.109.216101

Dobie, R., Wilson-Kanamori, J. R., Henderson, B. E. P., Smith, J. R., Matchett, K. P., Portman, J. R., et al. (2019). Single-Cell Transcriptomics Uncovers Zonation of Function in the Mesenchyme during Liver Fibrosis. Cell Rep 29, 1832-1847. e8. doi:10.1016/j.celrep.2019.10.024

Donohue, S. J., Roseboom, P. H., Illnerova, H., Weller, J. L., and Klein, D. C. (1993). Human Hydroxyindole-O-Methyltransferase: Presence of LINE-1 Fragment in a cDNA Clone and Pineal mRNA. DNA Cel Biol 12, 715-727. doi:10.1089/ dna.1993.12.715

Du, K., Hyun, J., Premont, R. T., Choi, S. S., Michelotti, G. A., Swiderska-Syn, M., et al. (2018). Hedgehog-YAP Signaling Pathway Regulates Glutaminolysis to Control Activation of Hepatic Stellate Cells. Gastroenterology 154, 1465-1479. e13. doi:10.1053/j.gastro.2017.12.022

Duffield, J. S. (2014). Cellular and Molecular Mechanisms in Kidney Fibrosis. J. Clin. Invest. 124, 2299-2306. doi:10.1172/JCI72267

Duffield, J. S., Lupher, M., Thannickal, V. J., and Wynn, T. A. (2013). Host Responses in Tissue Repair and Fibrosis. Annu. Rev. Pathol. 8, 241-276. doi:10.1146/annurev-pathol-020712-163930

Durán, W. N., and Sánchez, F. A. (2016). New Member of Endothelial Arsenal against Inflammation. Circ. Res. 119, 178-180. doi:10.1161/CIRCRESAHA.116.309122

Eming, S. A., Martin, P., and Tomic-Canic, M. (2014). Wound Repair and Regeneration: Mechanisms, Signaling, and Translation. Sci. Transl. Med. 6, 265sr6. doi:10.1126/scitranslmed.3009337

Fang, L., Chen, H., Kong, R., and Que, J. (2020). Endogenous Tryptophan Metabolite 5-Methoxytryptophan Inhibits Pulmonary Fibrosis by Downregulating the TGF-B/smad3 and PI3K/AKT Signaling Pathway. Life Sci. 260, 118399. doi:10.1016/j.lfs.2020.118399

Froese, A. R., Shimbori, C., Bellaye, P. S., Inman, M., Obex, S., Fatima, S., et al. (2016). Stretch-Induced Activation of Transforming Growth Factor-B1 in Pulmonary Fibrosis. Am. J. Respir. Crit. Care Med. 194, 84-96. doi:10.1164/ rccm.201508-1638OC 
Furukawa, F., Matsuzaki, K., Mori, S., Tahashi, Y., Yoshida, K., Sugano, Y., et al. (2003). p38 MAPK Mediates Fibrogenic Signal through Smad3 Phosphorylation in Rat Myofibroblasts. Hepatology 38, 879-889. doi:10.1053/jhep.2003.50384

Gibb, A. A., Lazaropoulos, M. P., and Elrod, J. W. (2020). Myofibroblasts and Fibrosis: Mitochondrial and Metabolic Control of Cellular Differentiation. Circ. Res. 127, 427-447. doi:10.1161/CIRCRESAHA.120.316958

Gonzalez-Gonzalez, F. J., Chandel, N. S., Jain, M., and Budinger, G. R. S. (2017). Reactive Oxygen Species as Signaling Molecules in the Development of Lung Fibrosis. Transl. Res. 190, 61-68. doi:10.1016/j.trsl.2017.09.005

Goodman, R. H., and Smolik, S. (2000). CBP/p300 in Cell Growth, Transformation, and Development. Genes Dev. 14, 1553-1577. Review. doi:10.1101/gad.14.13.1553

Grunstein, M. (1997). Histone Acetylation in Chromatin Structure and Transcription. Nature 389, 349-352. doi:10.1038/38664

Grynberg, K., Ma, F. Y., and Nikolic-Paterson, D. J. (2017). The JNK Signaling Pathway in Renal Fibrosis. Front. Physiol. 8, 829. doi:10.3389/fphys.2017.00829

Guo, L. Y., Yang, F., Peng, L. J., Li, Y. B., and Wang, A. P. (2020). CXCL2, a New Critical Factor and Therapeutic Target for Cardiovascular Diseases. Clin. Exp. Hypertens. 42, 428-437. doi:10.1080/10641963.2019.1693585

Hamid, T., Guo, S. Z., Kingery, J. R., Xiang, X., Dawn, B., and Prabhu, S. D. (2011). Cardiomyocyte NF-Kb P65 Promotes Adverse Remodelling, Apoptosis, and Endoplasmic Reticulum Stress in Heart Failure. Cardiovasc. Res. 89, 129-138. doi:10.1093/cvr/cvq274

Henderson, N. C., Rieder, F., and Wynn, T. A. (2020). Fibrosis: From Mechanisms to Medicines. Nature 587, 555-566. doi:10.1038/s41586-020-2938-9

Hernández-Gea, V., Ghiassi-Nejad, Z., Rozenfeld, R., Gordon, R., Fiel, M. I., Yue, Z., et al. (2012). Autophagy Releases Lipid that Promotes Fibrogenesis by Activated Hepatic Stellate Cells in Mice and in Human Tissues. Gastroenterology 142, 938-946. doi:10.1053/j.gastro.2011.12.044

Hidvegi, T., Ewing, M., Hale, P., Dippold, C., Beckett, C., Kemp, C., et al. (2010). An Autophagy-Enhancing Drug Promotes Degradation of Mutant Alpha1Antitrypsin Z and Reduces Hepatic Fibrosis. Science 329, 229-232. doi:10.1126/science.1190354

Hsu, H. S., Liu, C. C., Lin, J. H., Hsu, T. W., Hsu, J. W., Su, K., et al. (2017). Involvement of ER Stress, PI3K/AKT Activation, and Lung Fibroblast Proliferation in Bleomycin-Induced Pulmonary Fibrosis. Sci. Rep. 7, 14272. doi:10.1038/s41598-017-14612-5

Hsu, W. T., Tseng, Y. H., Jui, H. Y., Kuo, C. C., Wu, K. K., and Lee, C. M. (2021). 5Methoxytryptophan Attenuates Postinfarct Cardiac Injury by Controlling Oxidative Stress and Immune Activation. J. Mol. Cel. Cardiol. 158, 101-114. doi:10.1016/j.yjmcc.2021.05.014

Hu, B., Wu, Z., Liu, T., Ullenbruch, M. R., Jin, H., and Phan, S. H. (2007). Gut-Enriched Krüppel-Like Factor Interaction with Smad3 Inhibits Myofibroblast Differentiation. Am. J. Respir. Cel Mol Biol 36, 78-84. doi:10.1165/rcmb.2006-0043OC

$\mathrm{Hu}$, B., Wu, Z., Nakashima, T., and Phan, S. H. (2012). Mesenchymal-Specific Deletion of C/EBP $\beta$ Suppresses Pulmonary Fibrosis. Am. J. Pathol. 180, 2257-2267. doi:10.1016/j.ajpath.2012.02.010

Hu, X., Xu, Q., Wan, H., Hu, Y., Xing, S., Yang, H., et al. (2020). PI3K-Akt-mTOR/ PFKFB3 Pathway Mediated Lung Fibroblast Aerobic Glycolysis and Collagen Synthesis in Lipopolysaccharide-Induced Pulmonary Fibrosis. Lab. Invest. 100, 801-811. doi:10.1038/s41374-020-0404-9

Karlmark, K. R., Weiskirchen, R., Zimmermann, H. W., Gassler, N., Ginhoux, F., Weber, C., et al. (2009). Hepatic Recruitment of the Inflammatory Gr1+ Monocyte Subset Upon Liver Injury Promotes Hepatic Fibrosis. Hepatology 50, 261-274. doi:10.1002/hep.22950

Khomich, O., Ivanov, A. V., and Bartosch, B. (2019). Metabolic Hallmarks of Hepatic Stellate Cells in Liver Fibrosis. Cells 9, 24. doi:10.3390/cells9010024

Kingery, J. R., Hamid, T., Lewis, R. K., Ismahil, M. A., Bansal, S. S., Rokosh, G., et al. (2017). Leukocyte iNOS Is Required for Inflammation and Pathological Remodeling in Ischemic Heart Failure. Basic Res. Cardiol. 112, 19. doi:10.1007/s00395-017-0609-2

Kramann, R., DiRocco, D. P., and Humphreys, B. D. (2013). Understanding the Origin, Activation and Regulation of Matrix-Producing Myofibroblasts for Treatment of Fibrotic Disease. J. Pathol. 231, 273-289. doi:10.1002/path.4253

Krenkel, O., Hundertmark, J., Ritz, T. P., Weiskirchen, R., and Tacke, F. (2019). Single Cell RNA Sequencing Identifies Subsets of Hepatic Stellate Cells and Myofibroblasts in Liver Fibrosis. Cells 8, 503. doi:10.3390/cells8050503

Kumar, S., Boehm, J., and Lee, J. C. (2003). p38 MAP Kinases: Key Signalling Molecules as Therapeutic Targets for Inflammatory Diseases. Nat. Rev. Drug Discov. 2, 717-726. doi:10.1038/nrd1177
Kuppe, C., Ibrahim, M. M., Kranz, J., Zhang, X., Ziegler, S., Perales-Patón, J., et al. (2021). Decoding Myofibroblast Origins in Human Kidney Fibrosis. Nature 589, 281-286. doi:10.1038/s41586-020-2941-1

Lai, T. J., Wu, C. Y., Tsai, H. W., Lin, Y. M., and Sun, H. S. (2005). Polymorphism Screening and Haplotype Analysis of the Tryptophan Hydroxylase Gene (TPH1) and Association with Bipolar Affective Disorder in Taiwan. BMC Med. Genet. 6, 14. doi:10.1186/1471-2350-6-14

Leask, A., and Abraham, D. J. (2004). TGF-Beta Signaling and the Fibrotic Response. FASEB J. 18, 816-827. doi:10.1096/fj.03-1273rev

Li, Y., Liu, R., Wu, J., and Li, X. (2020). Self-Eating: Friend or Foe? the Emerging Role of Autophagy in Fibrotic Diseases. Theranostics 10, 7993-8017. doi:10.7150/thno.47826

Li, Y. H., Choi, D. H., Lee, E. H., Seo, S. R., Lee, S., and Cho, E. H. (2016). Sirtuin 3 (SIRT3) Regulates $\alpha$-Smooth Muscle Actin ( $\alpha$-SMA) Production through the Succinate Dehydrogenase-G Protein-Coupled Receptor 91 (GPR91) Pathway in Hepatic Stellate Cells. J. Biol. Chem. 291, 10277-10292. doi:10.1074/ jbc.M115.692244

Li, Y. H., Woo, S. H., Choi, D. H., and Cho, E. H. (2015). Succinate Causes a-SMA Production through GPR91 Activation in Hepatic Stellate Cells. Biochem. Biophys. Res. Commun. 463, 853-858. doi:10.1016/j.bbrc.2015.06.023

Luedde, T., and Schwabe, R. F. (2011). NF- $\kappa$ B in the Liver-Llinking Injury, Fibrosis and Hepatocellular Carcinoma. Nat. Rev. Gastroenterol. Hepatol. 8, 108-118. doi:10.1038/nrgastro.2010.213

Luo, K., and Lodish, H. F. (1996). Signaling by Chimeric Erythropoietin-TGF-Beta Receptors: Homodimerization of the Cytoplasmic Domain of the Type I TGFBeta Receptor and Heterodimerization with the Type II Receptor Are Both Required for Intracellular Signal Transduction. EMBO J. 15, 4485-4496. doi:10.1002/j.1460-2075.1996.tb00826.x

Ma, X. L., Kumar, S., Gao, F., Louden, C. S., Lopez, B. L., Christopher, T. A., et al. (1999). Inhibition of P38 Mitogen-Activated Protein Kinase Decreases Cardiomyocyte Apoptosis and Improves Cardiac Function after Myocardial Ischemia and Reperfusion. Circulation 99, 1685-1691. doi:10.1161/ 01.cir.99.13.1685

Manabe, I., Shindo, T., and Nagai, R. (2002). Gene Expression in Fibroblasts and Fibrosis: Involvement in Cardiac Hypertrophy. Circ. Res. 91, 1103-1113. doi:10.1161/01.res.0000046452.67724.b8

Mao, Y. Q., and Fan, X. M. (2015). Autophagy: A New Therapeutic Target for Liver Fibrosis. World J. Hepatol. 7, 1982-1986. doi:10.4254/wjh.v7.i16.1982

Martinez, F. J., Safrin, S., Weycker, D., Starko, K. M., Bradford, W. Z., King, T. E., Jr., et al. (2005). The Clinical Course of Patients with Idiopathic Pulmonary Fibrosis. Ann. Intern. Med. 142, 963-967. doi:10.7326/0003-4819-14212_part_1-200506210-00005

Melke, J., Goubran Botros, H., Chaste, P., Betancur, C., Nygren, G., Anckarsäter, H., et al. (2008). Abnormal Melatonin Synthesis in Autism Spectrum Disorders. Mol. Psychiatry 13, 90-98. doi:10.1038/sj.mp.4002016

Meng, X. M., Nikolic-Paterson, D. J., and Lan, H. Y. (2016). TGF- $\beta$ : The Master Regulator of Fibrosis. Nat. Rev. Nephrol. 12, 325-338. doi:10.1038/ nrneph.2016.48

Misharin, A. V., Morales-Nebreda, L., Reyfman, P. A., Cuda, C. M., Walter, J. M., McQuattie-Pimentel, A. C., et al. (2017). Monocyte-Derived Alveolar Macrophages Drive Lung Fibrosis and Persist in the Lung Over the Life Span. J. Exp. Med. 214, 2387-2404. doi:10.1084/jem.20162152

Molkentin, J. D., Bugg, D., Ghearing, N., Dorn, L. E., Kim, P., Sargent, M. A., et al. (2017). Fibroblast-Specific Genetic Manipulation of P38 Mitogen-Activated Protein Kinase In Vivo Reveals its Central Regulatory Role in Fibrosis. Circulation 136, 549-561. doi:10.1161/CIRCULATIONAHA.116.026238

Moris, D., Spartalis, M., Tzatzaki, E., Spartalis, E., Karachaliou, G. S., Triantafyllis, A. S., et al. (2017). The Role of Reactive Oxygen Species in Myocardial Redox Signaling and Regulation. Ann. Transl. Med. 5, 324. doi:10.21037/ atm.2017.06.17

Mouton, A. J., DeLeon-Pennell, K. Y., Rivera Gonzalez, O. J., Flynn, E. R., Freeman, T. C., Saucerman, J. J., et al. (2018). Mapping Macrophage Polarization Over the Myocardial Infarction Time Continuum. Basic Res. Cardiol. 113, 26. doi:10.1007/s00395-018-0686-x

Murray, L. A., Chen, Q., Kramer, M. S., Hesson, D. P., Argentieri, R. L., Peng, X., et al. (2011). TGF-Beta Driven Lung Fibrosis Is Macrophage Dependent and Blocked by Serum Amyloid P. Int. J. Biochem. Cel Biol. 43, 154-162. doi:10.1016/j.biocel.2010.10.013 
Noble, P. W., Barkauskas, C. E., and Jiang, D. (2012). Pulmonary Fibrosis: Patterns and Perpetrators. J. Clin. Invest. 122, 2756-2762. doi:10.1172/JCI60323

Olivetti, G., Quaini, F., Sala, R., Lagrasta, C., Corradi, D., Bonacina, E., et al. (1996). Acute Myocardial Infarction in Humans Is Associated with Activation of Programmed Myocyte Cell Death in the Surviving Portion of the Heart. J. Mol. Cel Cardiol. 28, 2005-2016. doi:10.1006/jmcc.1996.0193

Ong, S. B., Subrayan, S., Lim, S. Y., Yellon, D. M., Davidson, S. M., and Hausenloy, D. J. (2010). Inhibiting Mitochondrial Fission Protects the Heart against Ischemia/Reperfusion Injury. Circulation 121, 2012-2022. doi:10.1161/ CIRCULATIONAHA.109.906610

Pagan, C., Botros, H. G., Poirier, K., Dumaine, A., Jamain, S., Moreno, S., et al. (2011). Mutation Screening of ASMT, the Last Enzyme of the Melatonin Pathway, in a Large Sample of Patients with Intellectual Disability. BMC Med. Genet. 12, 17. doi:10.1186/1471-2350-12-17

Peyser, R., MacDonnell, S., Gao, Y., Cheng, L., Kim, Y., Kaplan, T., et al. (2019). Defining the Activated Fibroblast Population in Lung Fibrosis Using Single-Cell Sequencing. Am. J. Respir. Cel Mol. Biol 61, 74-85. doi:10.1165/rcmb.2018-0313OC

Prabhu, S. D., and Frangogiannis, N. G. (2016). The Biological Basis for Cardiac Repair after Myocardial Infarction: From Inflammation to Fibrosis. Circ. Res. 119, 91-112. doi:10.1161/CIRCRESAHA.116.303577

Rai, R., Verma, S. K., Kim, D., Ramirez, V., Lux, E., Li, C., et al. (2017). A Novel Acetyltransferase P300 Inhibitor Ameliorates Hypertension-Associated CardioRenal Fibrosis. Epigenetics 12, 1004-1013. doi:10.1080/15592294.2017.1370173

Rajasekaran, S., Reddy, N. M., Zhang, W., and Reddy, S. P. (2013). Expression Profiling of Genes Regulated by Fra-1/AP-1 Transcription Factor during Bleomycin-Induced Pulmonary Fibrosis. BMC Genomics 14, 381. doi:10.1186/1471-2164-14-381

Ramachandra, C. J. A., Hernandez-Resendiz, S., Crespo-Avilan, G. E., Lin, Y. H., and Hausenloy, D. J. (2020). Mitochondria in Acute Myocardial Infarction and Cardioprotection. EBioMedicine 57, 102884. doi:10.1016/j.ebiom.2020.102884

Ramachandran, P., Dobie, R., Wilson-Kanamori, J. R., Dora, E. F., Henderson, B. E. P., Luu, N. T., et al. (2019). Resolving the Fibrotic Niche of Human Liver Cirrhosis at Single-Cell Level. Nature 575, 512-518. doi:10.1038/s41586-019-1631-3

Ramachandran, P., Pellicoro, A., Vernon, M. A., Boulter, L., Aucott, R. L., Ali, A., et al. (2012). Differential Ly-6C Expression Identifies the Recruited Macrophage Phenotype, Which Orchestrates the Regression of Murine Liver Fibrosis. Proc. Natl. Acad. Sci. U. S. A. 109, E3186-E3195. doi:10.1073/pnas.1119964109

Reyfman, P. A., Walter, J. M., Joshi, N., Anekalla, K. R., McQuattie-Pimentel, A. C., Chiu, S., et al. (2019). Single-Cell Transcriptomic Analysis of Human Lung Provides Insights into the Pathobiology of Pulmonary Fibrosis. Am. J. Respir. Crit. Care Med. 199, 1517-1536. doi:10.1164/rccm.201712-2410OC

Schulien, I., Hockenjos, B., Schmitt-Graeff, A., Perdekamp, M. G., Follo, M., Thimme, R., et al. (2019). The Transcription Factor C-Jun/AP-1 Promotes Liver Fibrosis during Non-Alcoholic Steatohepatitis by Regulating Osteopontin Expression. Cell Death Differ 26, 1688-1699. doi:10.1038/s41418-018-0239-8

Sgalla, G., Kulkarni, T., Antin-Ozerkis, D., Thannickal, V. J., and Richeldi, L. (2019). Update in Pulmonary Fibrosis 2018. Am. J. Respir. Crit. Care Med. 200, 292-300. doi:10.1164/rccm.201903-0542UP

Stambe, C., Atkins, R. C., Tesch, G. H., Masaki, T., Schreiner, G. F., and NikolicPaterson, D. J. (2004). The Role of P38alpha Mitogen-Activated Protein Kinase Activation in Renal Fibrosis. J. Am. Soc. Nephrol. 15, 370-379. doi:10.1097/ 01.asn.0000109669.23650.56

Talman, V., and Ruskoaho, H. (2016). Cardiac Fibrosis in Myocardial InfarctionFrom Repair and Remodeling to Regeneration. Cell Tissue Res 365, 563-581. doi:10.1007/s00441-016-2431-9

Tang, P. M., Nikolic-Paterson, D. J., and Lan, H. Y. (2019). Macrophages: Versatile Players in Renal Inflammation and Fibrosis. Nat. Rev. Nephrol. 15, 144-158. doi:10.1038/s41581-019-0110-2

Thompson, P. R., Wang, D., Wang, L., Fulco, M., Pediconi, N., Zhang, D., et al. (2004). Regulation of the P300 HAT Domain via a Novel Activation Loop. Nat. Struct. Mol. Biol. 11, 308-315. doi:10.1038/nsmb740

Tong, M., Zheng, Q., Liu, M., Chen, L., Lin, Y. H., Tang, S. G., et al. (2021). 5Methoxytryptophan Alleviates Liver Fibrosis by Modulating FOXO3a/miR-21/ ATG5 Signaling Pathway Mediated Autophagy. Cell Cycle 20, 676-688. doi:10.1080/15384101.2021.1897241

Tsuchida, T., and Friedman, S. L. (2017). Mechanisms of Hepatic Stellate Cell Activation. Nat. Rev. Gastroenterol. Hepatol. 14, 397-411. doi:10.1038/nrgastro.2017.38

Turner, N. A., and Blythe, N. M. (2019). Cardiac Fibroblast P38 MAPK: A Critical Regulator of Myocardial Remodeling. J. Cardiovasc. Dev. Dis. 6, 27. doi:10.3390/jcdd6030027 van den Borne, S. W., Diez, J., Blankesteijn, W. M., Verjans, J., Hofstra, L., and Narula, J. (2010). Myocardial Remodeling after Infarction: The Role of Myofibroblasts. Nat. Rev. Cardiol. 7, 30-37. doi:10.1038/nrcardio.2009.199

Walther, D. J., Peter, J. U., Bashammakh, S., Hörtnagl, H., Voits, M., Fink, H., et al. (2003). Synthesis of Serotonin by a Second Tryptophan Hydroxylase Isoform. Science 299, 76. doi:10.1126/science.1078197

Wang, Y. F., Hsu, Y. J., Wu, H. F., Lee, G. L., Yang, Y. S., Wu, J. Y., et al. (2016). Endothelium-Derived 5-Methoxytryptophan Is a Circulating AntiInflammatory Molecule that Blocks Systemic Inflammation. Circ. Res. 119, 222-236. doi:10.1161/CIRCRESAHA.116.308559

Wernig, G., Chen, S. Y., Cui, L., Van Neste, C., Tsai, J. M., Kambham, N., et al. (2017). Unifying Mechanism for Different Fibrotic Diseases. Proc. Natl. Acad. Sci. U. S. A. 114, 4757-4762. doi:10.1073/pnas.1621375114

Wohlfahrt, T., Rauber, S., Uebe, S., Luber, M., Soare, A., Ekici, A., et al. (2019). PU.1 Controls Fibroblast Polarization and Tissue Fibrosis. Nature 566, 344-349. doi:10.1038/s41586-019-0896-x

Wouters, O. J., O’Donoghue, D. J., Ritchie, J., Kanavos, P. G., and Narva, A. S. (2015). Early Chronic Kidney Disease: Diagnosis, Management and Models of Care. Nat. Rev. Nephrol. 11, 491-502. doi:10.1038/nrneph.2015.85

Wu, K. K. (2021). Cytoguardin: A Tryptophan Metabolite against Cancer Growth and Metastasis. Int. J. Mol. Sci. 22, 4490. doi:10.3390/ijms22094490

Wu, K. K., Kuo, C. C., Yet, S. F., Lee, C. M., and Liou, J. Y. (2020). 5Methoxytryptophan: An Arsenal against Vascular Injury and Inflammation. J. Biomed. Sci. 27, 79. doi:10.1186/s12929-020-00671-w

Wuyts, W. A., Agostini, C., Antoniou, K. M., Bouros, D., Chambers, R. C., Cottin, V., et al. (2013). The Pathogenesis of Pulmonary Fibrosis: A Moving Target. Eur. Respir. J. 41, 1207-1218. doi:10.1183/09031936.00073012

Wynn, T. A., and Vannella, K. M. (2016). Macrophages in Tissue Repair, Regeneration, and Fibrosis. Immunity 44, 450-462. doi:10.1016/j.immuni.2016.02.015

Zepp, J. A., Zacharias, W. J., Frank, D. B., Cavanaugh, C. A., Zhou, S., Morley, M. P., et al. (2017). Distinct Mesenchymal Lineages and Niches Promote Epithelial Self-Renewal and Myofibrogenesis in the Lung. Cell 170, 1134-e10. e101. doi:10.1016/j.cell.2017.07.034

Zhang, F., Ayaub, E. A., Wang, B., Puchulu-Campanella, E., Li, Y. H., Hettiarachchi, S. U., et al. (2020a). Reprogramming of Profibrotic Macrophages for Treatment of Bleomycin-Induced Pulmonary Fibrosis. EMBO Mol. Med. 12, e12034. doi:10.15252/emmm.202012034

Zhang, J., Wang, Y. T., Miller, J. H., Day, M. M., Munger, J. C., and Brookes, P. S. (2018). Accumulation of Succinate in Cardiac Ischemia Primarily Occurs via Canonical Krebs Cycle Activity. Cel Rep 23, 2617-2628. doi:10.1016/j.celrep.2018.04.104

Zhang, X. L., Xing, R. G., Chen, L., Liu, C. R., and Miao, Z. G. (2016). PI3K/Akt Signaling Is Involved in the Pathogenesis of Bleomycin-Induced Pulmonary Fibrosis via Regulation of Epithelial-mesenchymal Transition. Mol. Med. Rep. 14, 5699-5706. doi:10.3892/mmr.2016.5960

Zhang, Y., Murugesan, P., Huang, K., and Cai, H. (2020b). NADPH Oxidases and Oxidase Crosstalk in Cardiovascular Diseases: Novel Therapeutic Targets. Nat. Rev. Cardiol. 17, 170-194. doi:10.1038/s41569-019-0260-8

Zhu, J., Wu, J., Frizell, E., Liu, S. L., Bashey, R., Rubin, R., et al. (1999). Rapamycin Inhibits Hepatic Stellate Cell Proliferation In Vitro and Limits Fibrogenesis in an In Vivo Model of Liver Fibrosis. Gastroenterology 117, 1198-1204. doi:10.1016/s0016-5085(99)70406-3

Conflict of Interest: The author declares that the research was conducted in the absence of any commercial or financial relationships that could be construed as a potential conflict of interest.

Publisher's Note: All claims expressed in this article are solely those of the authors and do not necessarily represent those of their affiliated organizations, or those of the publisher, the editors, and the reviewers. Any product that may be evaluated in this article, or claim that may be made by its manufacturer, is not guaranteed or endorsed by the publisher.

Copyright $\odot 2021 \mathrm{Wu}$. This is an open-access article distributed under the terms of the Creative Commons Attribution License (CC BY). The use, distribution or reproduction in other forums is permitted, provided the original author(s) and the copyright owner(s) are credited and that the original publication in this journal is cited, in accordance with accepted academic practice. No use, distribution or reproduction is permitted which does not comply with these terms. 